\title{
Treatment of Electrical Storm with Amiodarone in Brugada Syndrome- an Unexpected Protective Effect
}

Novak $\mathrm{J}^{1,2}$ and Lambiase $P D^{1 *}$

${ }^{1}$ The Heart Hospital, UCH, London, United Kingdom

${ }^{2}$ Bürgerspital Solothurn, Solothurn, Switzerland

\begin{abstract}
We are reporting on a 53 year old man with proven Brugada syndrome and ICD implantation for resuscitation in context of polymorphic VT. After recurrent arrhythmia he was treated with Amiodarone. This showed to have a protective effect despite various reports suggesting avoiding Amiodarone in Brugada syndrome.
\end{abstract}

Keywords: Brugada syndrome; Genetic disorders

\section{Case}

A 53 year old man with a history of EEG proven epilepsy on treatment with Levetiracetam was successfully resuscitated from cardiac arrest due to polymorphic VT in January 2013. Resting ECG shows RBBB pattern. Ajmaline challenge testing induced typical type I coved ST elevation in V1-3 diagnostic of Brugada Syndrome with polymorphic VE's and couplets (Figure 1). Cardiac magnetic resonance imaging revealed only a slightly hypokinetic apex consistent with post cardiac arrest changes with normal right \& left ventricular size and function, no late enhancement/fat infiltration. A single chamber, BSCI Incepta Internal Cardiac Defibrillator (ICD) was implanted. Defibrillation testing achieved successful termination of induced Ventricular Fibrillation $10 \mathrm{~J}$ below maximum energy. The ICD was programmed to VVI mode back-up pacing $30 \mathrm{bpm}$, VF therapy Zone with shock and ATP during charging above 220/min and patient discharged home on Levetiracetam.

Two days later the patient was admitted to his local hospital with 9 appropriate ICD shocks- stored EGMs confirmed sudden onset polymorphic tachycardia mainly at 250-310 bpm terminated with 41 J shock (Figure 2). Amiodarone was commenced in his local hospital, pacing was reprogrammed to VVI $90 \mathrm{bpm}$ and the patient was discharged. Since amiodarone is reported to be pro-arrythmogenic in patients having Brugada syndrome [1-3], we elected to stop amiodarone
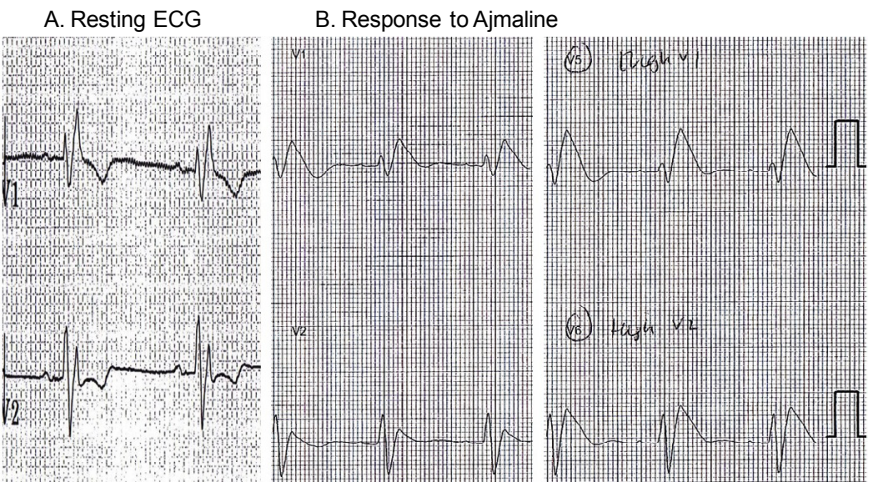

Standard Leads

High Leads

Figure 1A: Resting ECG on presentation-showing right bundle branch block pattern in leads V1-V3. B: Response to ajmaline challenge. Coved ST elevation standard leads V1 \& V2 as well as high chest leads in $3^{\text {rd }}$ intercostal space.

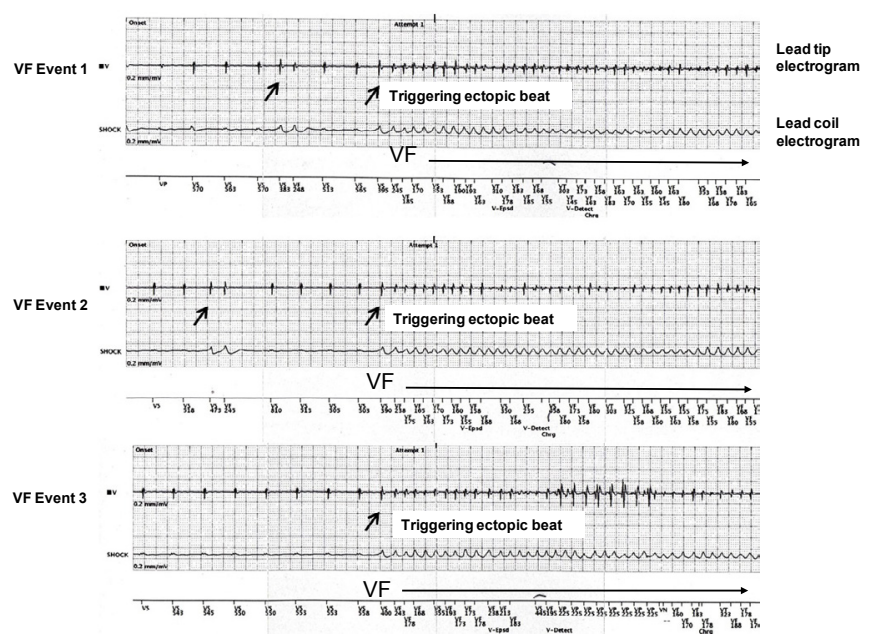

Figure 2: ICD Electrogram traces of 3 VF Events. Triggering ventricular ectopics trigger VF at critically timed intervals. The ectopics are highlighted by an arrow.

2 weeks subsequently and replace this with Quinidine to protect the patient against further episodes of arrhythmia.

The patient was admitted for the initiation of Quinidine, having stopped amiodarone for 2 days. The device check revealed that since stopping amiodarone the day prior to admission there has been 13 episodes of NSVT, longest 11 beats, which all were self terminating, no therapies delivered. These episodes were all triggered by closely coupled premature ventricular beats. As amiodarone appeared to be successfully suppressing VT, it was restarted (200 mg once a day) and

*Corresponding author: Pier D. Lambiase, PhD FRCP, Reader and Consultant Cardiologist, The Heart Hospital, University College Hospital \& Institute of Cardiovascular Sciences, UCL, 16-18 Westmoreland Street, London W1G 8PH., UK, Tel: 0044207573 8888; Fax: 0044207573 8847; E-mail: pier.lambiase@uclh.nhs.uk

Received July 09, 2013; Accepted September 10, 2013; Published September 18,2013

Citation: Novak J, Lambiase PD (2013) Treatment of Electrical Storm with Amiodarone in Brugada Syndrome- an Unexpected Protective Effect. J Genet Syndr Gene Ther 4: 176. doi:10.4172/2157-7412.1000176

Copyright: ( 2013 Novak J, et al. This is an open-access article distributed under the terms of the Creative Commons Attribution License, which permits unrestricted use, distribution, and reproduction in any medium, provided the original author and source are credited. 
initially the base rate programmed to ventricular pacing (VVI) 80 beats/ min and the patient discharged. On subsequent follow-ups over the next month the device checks showed no arrhythmia despite marked coved ST elevation+ during treatment on resting ECG and the base pacing rate was reduced to $45 \mathrm{bpm}$. The patient continued on treatment with Amiodarone $200 \mathrm{mg}$ od. Despite previous reports indicating that Amiodarone should be avoided in Brugada Syndrome this patient was successfully treated to prevent episodes of ventricular arrhythmia with this drug.

\section{Discussion}

\section{Background}

Brugada syndrome is characterised by coved-type ST-segment elevation followed by a negative $\mathrm{T}$-wave in right precordial leads V1-V3 (type 1 ECG pattern) or saddleback pattern in V1 (with STsegment elevation) or V2 (without ST-segment elevation), in addition to either documented ventricular fibrillation, polymorphic ventricular tachycardia, family history of sudden cardiac death aged $<45$ years, coved-type ECG changes in family members, inducibility of VT with programmed electrical stimulation, syncope or nocturnal agonal respiration. The estimated prevalence is $1 / 2000$ in Caucasians although it may be greater in individuals of Asian descent.

It is inherited in an autosomal dominant mode of transmission, with several possible genotypes identified coding calcium channels (CACNA1C, CACNB2b, CACNA2D1) resulting in loss of function of basal L-type calcium current (ICa,L), sodium channels leading to loss of function of $\mathrm{INa}$ (SCN1B, SCN3B) as well gain of function of potassium currents (KCNE3, KCND3,KCNE5). To date, mutations in at least 6 distinct susceptibility genes have been identified (Table 1). The most common being mutations in the SCN5A gene (15-30\% cases).

A vast number of mutations ( $>100$ known, missense mutations, nonsense mutation, nucleotide insertion/deletion and splice site mutation) occurring in the SCN5A gene account for roughly $30 \%$ of all patients, coding the alpha subunit of subunit of the cardiac sodium channel, representing the commonest mutation in Brugada syndrome. It causes loss of function of the sodium channel (decreased expression of Nav1.5 proteins in sarcolemma and expression of non-functional or altered channels diminishing fast upstroke (Phase 0 ) of the action potential) leading to slowing of conduction (delayed activation, earlier or faster inactivation, enhanced slow inactivation and delayed recovery from inactivation). As these channels play a key role in electrical cardiac function SCN5A mutations not only cause Brugada syndrome but there is a significant overlap to other electrical or structural cardiac diseases (Long QT syndrome type 3, progressive cardiac conduction disease, sick sinus syndrome, atrial fibrillation, atrial standstill or dilated cardiomyopathy) [4]. Genetic testing is useful especially in context of family screening in case the index patient is gene positive. In this case there is no remarkable family history of SCD, unfortunately until now the family refused to undergo further functional or genetic testing. As the diagnosis was clear in this patient and family screening is currently not an option, gene testing has not been carried out. Yet as this patient showed a remarkable and unexpected response to Amiodarone in context of obvious Brugada syndrome blood has been taken during follow up for gene sequencing, still these results were pending when this article has been published, thus we will report and discuss these results separately in an addendum as soon as genes are available.

This mechanism of coved ST elevation on the ECG is debated with 2 hypotheses having been proposed-the repolarisation \& depolarisation hypotheses repectively [5]. According to the repolarisation hypothesis, in contrast to endocardium, the epicardial action potential (AP) has a prominent phase 1 resulting in a spike and dome morphology (Figure 3 ). This is thought to be the result of a more pronounced expression of the transient outward potassium channel $\left(\mathrm{I}_{\mathrm{TO}}\right)$ in epicardial cells, largely responsible for the rapid downstroke of phase 1 . If, for any reason there is a reduction in the amplitude of phase 0 , this will result in a lower voltage level at which phase 1 begins. This affects the activation/ inactivation kinetics of both $\mathrm{I}_{\mathrm{TO}}$ and the availability of the L-type calcium channel involved in the phase 2 plateau, resulting in a loss of the AP dome and consequent shortening of the epicardial AP duration (APD). The less pronounced phase 1 downstroke of endocardium due to lower $\mathrm{I}_{\mathrm{TO}}$ expression allows for conservation of the AP pattern, even with some reduction in phase 0 amplitude. The transmural gradient during phase 1 between the epicardium and endocardium was shown in the canine wedge experiments by Antzelevitch and Yan, to contribute to both J point and ST elevation on the surface ECG [6].

The depolarisation hypothesis proposes that coved ST elevation develops as a result of delays in conduction between the right ventricular body and outflow tract (RVOT). If the RVOT action potential is delayed in relation to that of the RV body, a voltage gradient develops between the two sites with the more positive membrane potential in the RV body (source) driving intercellular current to the RVOT (sink). The circuit is completed by extracellular current from the RVOT to the RV, inscribing a positive deflection on the ECG (J point and ST elevation). Conduction delays in the RVOT have been demonstrated in a number of human studies in BrS to support this hypothesis $[7,8]$.

\begin{tabular}{|c|c|c|}
\hline $\begin{array}{l}\text { Brugada } \\
\text { syndrome }\end{array}$ & $\begin{array}{l}\text { SCN5A } \\
\text { GPD1L } \\
\text { CACNA1C } \\
\text { CACNB2B SCN1B } \\
\text { KCNE3 } \\
\text { SCN3B } \\
\text { HCN4 }\end{array}$ & $\begin{array}{l}I_{\mathrm{Na}} \text { sodium channel } \alpha \text {-subunit } \\
\mathrm{Glycerol}-3 \text {-phosphate dehydrogenase } 1 \text { like } \\
I_{\mathrm{Ca}, \mathrm{L}} \text { calcium channel } \alpha \text {-subunit } \\
I_{\mathrm{I}} \text { calcium channel } \beta \text {-subunit } \\
I_{\mathrm{Na}} \text { sodium channel beta- } 1 \text { subunit } \\
I_{\text {to }} \text { voltage-gated potassium channel, Isk-related family } \\
I_{\mathrm{Na}} \text { sodium channel type III beta } \\
I_{f} \text { hyperpolarization-activated potassium channel } 4\end{array}$ \\
\hline
\end{tabular}

Table 1: Genes Responsible for Brugada Syndrome.

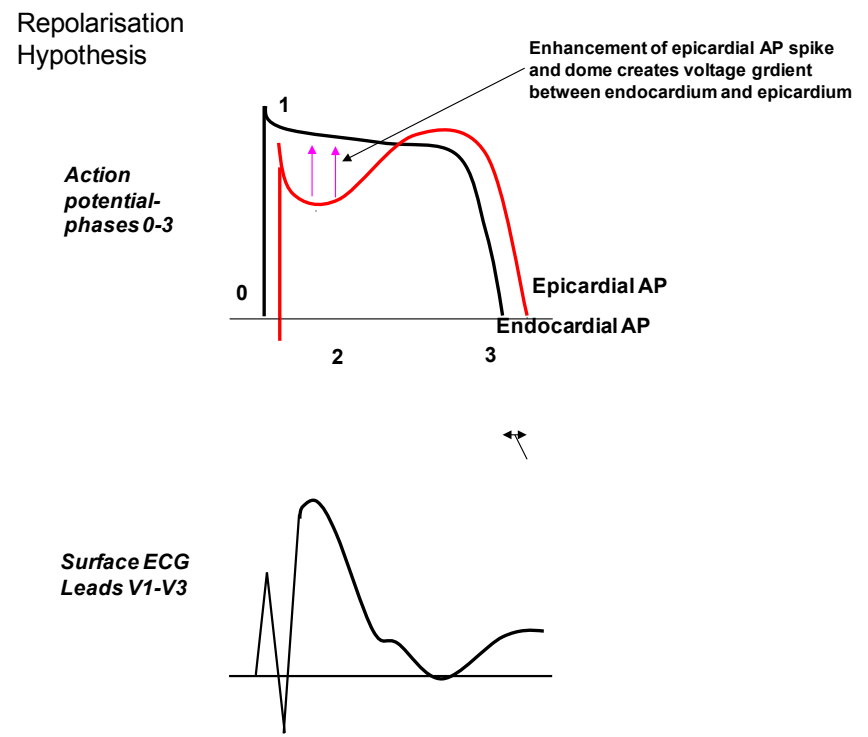

Figure 3: Repolarisation Hypothesis. Illustration of the proposed epicardial and endocardial electrogram morphologies creating the voltage gradients forming the coved ST elevation typical of the type 1 Brugada ECG. 
Citation: Novak J, Lambiase PD (2013) Treatment of Electrical Storm with Amiodarone in Brugada Syndrome- an Unexpected Protective Effect. J Genet Syndr Gene Ther 4: 176. doi:10.4172/2157-7412.1000176

\section{Mechanism of VF}

In this case, VF was consistently initiated by a closely coupled premature ventricular contraction (PVC).

VF in Brugada Syndrome is thought to be initated by phase 2 reentry in the RVOT $[9,10]$. Previous studies (mainly experimental) suggested that in patients with Brugada syndrome the large transmural repolarisation gradient in the RVOT facilitates current flow during phase 2 of the action potential from the endoicardium to epicardial layers-hence the phenomenon of phase 2 re-entry due to the difference in repolarisation times between the mayocardial layers in the RVOT.

The initiation of VF is illustrated on the ICD tracings which show the triggering beats repeatedly occurred at a coupling interval (CI) of $240 \mathrm{~ms}$. Recent studies of Brugada syndrome demonstrated that the CI of the triggered PVC of VF was within the range of 336-453 ms and the onset of the PVC was close to the end of $\mathrm{T}$ wave of the preceding beat which is consistent with the timing of phase 2 re-entry triggering beats $[11,12]$. Nam also reported that the CI of a triggered beat in patients with early repolarisation J point elevation pattern on ECG tended to be shorter than that seen in patients with Brugada syndrome [10]. In addition, Viskin et al. reported that the CI of a triggered beat was $302 \pm$ $52 \mathrm{~ms}$ in 22 patients with idiopathic VF. In our patient, the triggering beats tended to be shorter and similar to the short-coupling variant form of idiopathic VF as opposed to the later PVC's reported in $\mathrm{BrS}$ [13].

\section{Amiodarone in brugada syndrome}

Amiodarone is predominantly a potassium ion channel-blocking agent (Vaughan Williams class III) but has been shown in vitro to have sodium ion channel-blocking properties, especially in the acute phase of its administration [14,15]. There have been limited case reports in the literature demonstrating how amiodarone can unmask the Brugada ECG and trigger ventricular arrhythmia presumably by enhancing the $\mathrm{Na}$ channel blocking effect in ion channel mutation carriers. What is unusual in this case is the fact that although the amiodarone promoted the type 1 ECG changes of Brugada Syndrome directly affecting the substrate, it suppressed the ventricular ectopic triggers leading to VF. The fact the PVCs were early coupled beats would suggest that the alternative anti-arrhythmic actions of the agent were preventing triggered activity as opposed to phase 2 re-entry. Since amiodarone significantly prolongs action potential during and increases refractoriness, it is conceivable that even if phase 2 re-entry were initiated the myocardium was too refractory to perpetuate it, since the patient had an ICD in situ, he had adequate protection to cardiovert VF should it occur. Utilisation of amiodarone for the purpose of suppressing VF in this circumstance should not be considered first line but given its efficacy in this case, it would suggest it may have a role in certain cirumstances e.g. When quinidine the $\mathrm{I}_{\mathrm{T}}$ channel blocker recognised to suppress VF storm in Brugada Syndrome is not available or contraindicated due to side effects (liver dysfunction, blood dyscrasia). There is an emerging role of utilisation of epicardial

This article was originally published in a special issue, Genetic Disorders handled by Editor(s). Dr. Xiaobo Li, Yeshiva University, USA ablation to modify the substrate in cases of VF storm due to Brugada syndrome which may evolve as the first line therapy in recurrent VF cases as opposed to pharmacological suppression depending upon its long term success and applicability [16].

\section{References}

1. D'Aloia A, Vizzardi E, Bugatti S, Zanini G, Bontempi L, et al. (2012) Brugada syndrome phenotype cardiac arrest in a young patient unmasked during the acute phase of amiodarone infusion: disclosure and aggravation of Brugada electrocardiographic pattern. J Electrocardiol 45: 411-413.

2. Joshi S, Raiszadeh F, Pierce W, Steinberg JS (2007) Antiarrhythmic induced electrical storm in Brugada syndrome: a case report. Ann Noninvasive Electrocardiol 12: 274-278.

3. Nägele H, Behrens S, Castel A (2008) Ventricular tachycardia and aggravation of Brugada ECG pattern in a patient with coronary artery disease and combined amiodarone and betablocker therapy. Clin Res Cardiol 97: 56-60.

4. Mizusawa Y, Wilde AA (2012) Brugada syndrome. Circ Arrhythm Electrophysiol 5: 606-616.

5. Meregalli PG, Wilde AA, Tan HL (2005) Pathophysiological mechanisms of Brugada syndrome: depolarization disorder, repolarization disorder, or more? Cardiovasc Res 67: 367-378.

6. Yan GX, Antzelevitch C (1996) Cellular basis for the electrocardiographic J wave. Circulation 93: 372-379.

7. Postema PG, van Dessel PF, Kors JA, Linnenbank AC, van Herpen G, et al (2010) Local depolarisation abnormalities are the dominant pathophysiologic mechanism for type 1 electrocardiogram in Brugada syndrome. J Am Coll Cardiol 55: 789-797.

8. Lambiase PD, Ahmed AK, Ciaccio EJ, Brugada R, Lizotte E, et al. (2009) Highdensity substrate mapping in Brugada syndrome: combined role of conduction and repolarization heterogeneities in arrhythmogenesis. Circulation 120: 106$117,1-4$.

9. Brugada P, Brugada J (1992) Right bundle branch block, persistent ST segmen elevation and sudden cardiac death: a distinct clinical and electrocardiographic syndrome. J Am Coll Cardiol 20: 1391-1396.

10. Wilde AA, Antzelevitch C, Borggrefe M, Brugada J, Brugada R, et al. (2002) Proposed diagnostic criteria for the Brugada syndrome: consensus report Circulation 106: 2514-2519.

11. Nam GB, Ko KH, Kim J, Park KM, Rhee KS, et al. (2010) Mode of onset of ventricular fibrillation in patients with early repolarization pattern vs. Brugada syndrome. Eur Heart J 31: 330-339.

12. Kakishita M, Kurita T, Matsuo K, Taguchi A, Suyama K, et al. (2000) Mode of onset of ventricular fibrillation in patients with Brugada syndrome detected by implantable cardioverter defibrillator therapy. J Am Coll Cardiol 36: 1646-1653.

13. Viskin S, Lesh MD, Eldar M, Fish R, Setbon I, et al. (1997) Mode of onse of malignant ventricular arrhythmias in idiopathic ventricular fibrillation. J Cardiovasc Electrophysiol 8: 1115-1120.

14. Sheldon RS, Hill RJ, Cannon NJ, Duff HJ (1989) Amiodarone: biochemical evidence for binding to a receptor for class I drugs associated with the rat cardiac sodium channel. Circ Res 65: 477-482.

15. Lalevée N, Nargeot J, Barrére-Lemaire S, Gautier P, Richard S (2003) Effects of amiodarone and dronedarone on voltage-dependent sodium current in human cardiomyocytes. J Cardiovasc Electrophysiol 14: 885-890.

16. Nademanee K, Veerakul G, Chandanamattha P, Chaothawee $L$ Ariyachaipanich A, et al. (2011) Prevention of ventricular fibrillation episodes in Brugada syndrome by catheter ablation over the anterior right ventricular outflow tract epicardium. Circulation 123: 1270-1279. 